\title{
Crystal structure of bis(diaquamanganese(II))hexaacetatomanganese(II) octahydrate, $\left(\left(\mathrm{H}_{2} \mathrm{O}\right)_{2} \mathrm{Mn}\right)_{2}\left(\mathrm{C}_{2} \mathrm{H}_{3} \mathrm{O}_{2}\right)_{6} \mathrm{Mn} \cdot 8 \mathrm{H}_{2} \mathrm{O}$
}

\author{
S.-Y. Li ${ }^{\mathrm{I}}$, Y.-H. Li', H.-L. Liu', H.-L. Zhu*,I and D.-Q. Wang ${ }^{\mathrm{II}}$ \\ I Wuhan Institute of Science and Technology, Department of Environmental and Chemical Engineering, Wuhan, 430073 P. R. China \\ II Liaocheng University, Department of Chemistry, Liaocheng, 252059 P. R. China
}

Received March 17, 2003, accepted in revised form and available on-line July 30, 2003; CCDC-No. 1267/1069

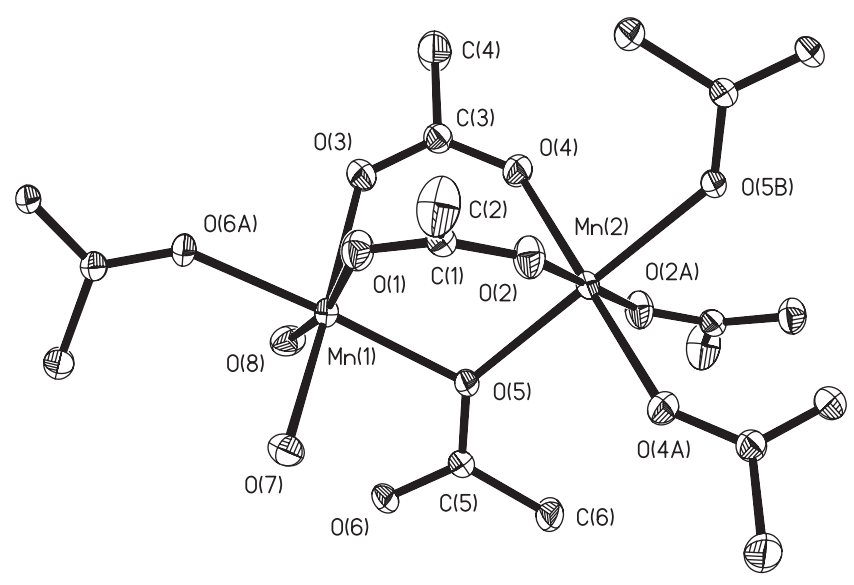

Abstract

$\mathrm{C}_{12} \mathrm{H}_{42} \mathrm{Mn}_{3} \mathrm{O}_{24}$, monoclinic, $P 12{ }_{1} / c 1$ (No. 14), $a=10.409(8) \AA$, $b=17.52(1) \AA, c=9.059(7) \AA, \beta=110.93(1)^{\circ}, V=1543.3 \AA^{3}$, $Z=2, R_{\mathrm{gt}}(F)=0.032, w R_{\mathrm{ref}}\left(F^{2}\right)=0.089, T=298 \mathrm{~K}$.

\section{Source of material}

Commercial $\mathrm{Mn}(\mathrm{OAc})_{2} \cdot 4 \mathrm{H}_{2} \mathrm{O}(\mathrm{OAc}=$ acetate anion $)$ was dissolved to anhydrous methanol solution, to which diethyl ether was diffused for three days. Large pink plate-like crystals were precipitated, filtered and washed with methanol for three times, and dried in a vacuum under $\mathrm{CaCl}_{2}$. Elemental analysis: found $-\mathrm{C}$, $19.81 \%$; H, 5.88\% ; calc. for $\mathrm{C}_{4} \mathrm{H}_{14} \mathrm{MnO}_{8}-\mathrm{C}, 19.60 \% ; \mathrm{H}, 5.76 \%$.

\section{Experimental setails}

Because the $\mathrm{H}$ atoms attached to the water molecules were not able to be calculated theoretically, these hydrogen atoms were located from difference Fourier maps and refined isotropically.

\section{Discussion}

The title complex is a three-dimensional polymeric one. There are two kinds of univalent $\mathrm{Mn}$ (II) atoms in each trinuclear Mn unit. $\mathrm{Mn}(1)$ atom in the complex is six-coordinated to six oxygen atoms from four different acetate anions and two water molecules, forming a distorted octahedron (the diagonal angles are $168.79(7)^{\circ}, 170.22(7)^{\circ}$ and $177.09(6)^{\circ}$. $\mathrm{Mn}(2)$ is surrounded by six oxygen atoms from six different acetate anions also to constitute a distorted octahedron (the diagonal angles are 180.00(9) ${ }^{\circ}$, $180.0(1)^{\circ}$ and $\left.180.00(3)^{\circ}\right)$. $\mathrm{Mn}(1)$ and $\mathrm{Mn}(2)$ atoms are bridged together by three different acetate anion ligands, two of which adopt dinucleated $O, O^{\prime}$-bridged coordination mode to ligate to the two metal atoms, and the third acetate ligand is in $O, O, O^{\prime}$ bridged mode to three $\mathrm{Mn}$ atoms. Each of the donor oxygen atoms in the acetates with $O, O, O^{\prime}$-bridged mode coordinates to another trimer manganese unit, which connects the complex to form a two-dimensional network along $b c$-plane. Uncoordinated water molecules join the neighboring planes by hydrogen bonds to form a three-dimensional network, in which small tunnels with the diameter of about $4 \AA$ are regularly distributed.

Table 1. Data collection and handling.
Crystal:

Wavelength:

$\mu$ :

Diffractometer, scan mode:

$2 \theta_{\max }$ :

$N(h k l)_{\text {measured }} N(h k l)_{\text {unique: }}$

Criterion for $I_{\mathrm{obs}}, N(h k l)_{\mathrm{gt}}$ :

$N(\text { param })_{\text {refined: }}$

Programs: pink plate, size $0.20 \times 0.33 \times 0.52 \mathrm{~mm}$

Mo $K_{\alpha}$ radiation $(0.71073 \AA)$

$12.97 \mathrm{~cm}^{-1}$

Bruker SMART CCD, $\varphi / \omega$

$52.76^{\circ}$

8657,3098

$I_{\mathrm{obs}}>2 \sigma\left(I_{\mathrm{obs}}\right), 2423$

226

SHELXTL [1], SHELXTL-plus [2]
Table 2. Atomic coordinates and displacement parameters (in $\AA^{2}$ ).

\begin{tabular}{llllll}
\hline Atom & Site & $x$ & $y$ & $z$ & $U_{\text {iso }}$ \\
\hline $\mathrm{H}(2 \mathrm{~A})$ & $4 e$ & -0.3544 & 0.0274 & 0.1649 & 0.088 \\
$\mathrm{H}(2 \mathrm{~B})$ & $4 e$ & -0.3891 & 0.1145 & 0.1376 & 0.088 \\
$\mathrm{H}(2 \mathrm{C})$ & $4 e$ & -0.2918 & 0.0855 & 0.3036 & 0.088 \\
$\mathrm{H}(4 \mathrm{~A})$ & $4 e$ & 0.3649 & 0.1086 & 0.5169 & 0.095 \\
$\mathrm{H}(4 \mathrm{~B})$ & $4 e$ & 0.4136 & 0.0447 & 0.4275 & 0.095 \\
$\mathrm{H}(4 \mathrm{C})$ & $4 e$ & 0.3119 & 0.0247 & 0.5144 & 0.095 \\
$\mathrm{H}(6 \mathrm{~A})$ & $4 e$ & -0.0049 & 0.0363 & -0.3229 & 0.073 \\
$\mathrm{H}(6 \mathrm{~B})$ & $4 e$ & 0.0843 & 0.0914 & -0.3834 & 0.073 \\
$\mathrm{H}(6 \mathrm{C})$ & $4 e$ & -0.0762 & 0.0995 & -0.4477 & 0.073 \\
$\mathrm{H}(1)$ & $4 e$ & $-0.162(3)$ & $0.282(1)$ & $-0.174(2)$ & $0.050(9)$ \\
$\mathrm{H}(2)$ & $4 e$ & $-0.228(3)$ & $0.270(2)$ & $-0.064(4)$ & $0.08(1)$ \\
$\mathrm{H}(3)$ & $4 e$ & $0.193(3)$ & $0.285(1)$ & $-0.026(2)$ & $0.053(9)$ \\
$\mathrm{H}(4)$ & $4 e$ & $0.274(3)$ & $0.275(2)$ & $0.133(3)$ & $0.08(1)$ \\
$\mathrm{H}(5)$ & $4 e$ & $0.725(3)$ & $0.157(2)$ & $0.808(5)$ & $0.12(2)$ \\
$\mathrm{H}(6)$ & $4 e$ & $0.701(4)$ & $0.077(1)$ & $0.783(6)$ & $0.12(2)$ \\
$\mathrm{H}(7)$ & $4 e$ & $0.411(6)$ & $0.360(2)$ & $0.339(6)$ & $0.15(2)$ \\
$\mathrm{H}(8)$ & $4 e$ & $0.478(5)$ & $0.288(3)$ & $0.380(5)$ & $0.19(3)$ \\
$\mathrm{H}(9)$ & $4 e$ & $0.546(4)$ & $0.270(2)$ & $0.929(6)$ & $0.13(2)$ \\
$\mathrm{H}(10)$ & $4 e$ & $0.602(9)$ & $0.326(5)$ & $1.046(9)$ & $0.43(7)$ \\
$\mathrm{H}(11)$ & $4 e$ & $0.706(5)$ & $0.903(3)$ & $1.097(2)$ & $0.15(3)$ \\
$\mathrm{H}(12)$ & $4 e$ & $0.729(5)$ & $0.899(4)$ & $0.954(6)$ & $0.23(4)$ \\
& & & & &
\end{tabular}

* Correspondence author (e-mail: hlzhu@wist.edu.cn) 
Table 3. Atomic coordinates and displacement parameters (in $\AA^{2}$ ).

\begin{tabular}{|c|c|c|c|c|c|c|c|c|c|c|}
\hline Atom & Site & $x$ & $y$ & $z$ & $U_{11}$ & $U_{22}$ & $U_{33}$ & $U_{12}$ & $U_{13}$ & $U_{23}$ \\
\hline $\operatorname{Mn}(1)$ & $4 e$ & $0.02019(4)$ & $0.20172(2)$ & $0.08744(4)$ & $0.0335(2)$ & $0.0203(2)$ & $0.0263(2)$ & $0.0006(1)$ & $0.0105(2)$ & $-0.0029(1)$ \\
\hline $\operatorname{Mn}(2)$ & $2 a$ & 0 & 0 & 0 & $0.0283(3)$ & $0.0170(2)$ & $0.0270(3)$ & $-0.0001(2)$ & $0.0092(2)$ & $0.0006(2)$ \\
\hline $\mathrm{O}(1)$ & $4 e$ & $-0.1370(2)$ & $0.15178(9)$ & $0.1677(2)$ & $0.047(1)$ & $0.0309(9)$ & $0.045(1)$ & $-0.0078(8)$ & $0.0248(9)$ & $-0.0056(8)$ \\
\hline $\mathrm{O}(2)$ & $4 e$ & $-0.1638(2)$ & $0.03336(9)$ & $0.0780(2)$ & $0.042(1)$ & $0.0310(9)$ & $0.051(1)$ & $-0.0009(8)$ & $0.0263(9)$ & $-0.0058(8)$ \\
\hline $\mathrm{O}(3)$ & $4 e$ & $0.1744(2)$ & $0.14712(9)$ & $0.2862(2)$ & $0.047(1)$ & $0.0288(9)$ & $0.037(1)$ & $0.0075(8)$ & $0.0062(8)$ & $0.0015(8)$ \\
\hline $\mathrm{O}(4)$ & $4 e$ & $0.1714(2)$ & $0.02628(9)$ & $0.2129(2)$ & $0.041(1)$ & $0.0322(9)$ & $0.034(1)$ & $-0.0010(8)$ & $0.0018(8)$ & $-0.0044(8)$ \\
\hline $\mathrm{O}(6)$ & $4 e$ & $0.0244(2)$ & $0.21093(8)$ & $-0.2453(2)$ & $0.052(1)$ & $0.0215(8)$ & $0.032(1)$ & $-0.0009(7)$ & $0.0169(8)$ & $0.0050(7)$ \\
\hline $\mathrm{O}(7)$ & $4 e$ & $-0.1621(2)$ & $0.2537(1)$ & $-0.0946(2)$ & $0.038(1)$ & $0.038(1)$ & $0.035(1)$ & $0.0078(8)$ & $0.0163(8)$ & $0.0075(8)$ \\
\hline $\mathrm{O}(8)$ & $4 e$ & $0.2047(2)$ & $0.2548(1)$ & $0.0561(2)$ & $0.043(1)$ & $0.046(1)$ & $0.033(1)$ & $-0.0107(9)$ & $0.0117(9)$ & $0.0002(9)$ \\
\hline $\mathrm{O}(9)$ & $4 e$ & $0.6676(2)$ & $0.1221(1)$ & $0.7560(3)$ & $0.048(1)$ & $0.048(1)$ & $0.054(1)$ & $0.002(1)$ & $0.008(1)$ & $0.003(1)$ \\
\hline $\mathrm{O}(10)$ & $4 e$ & $0.4280(2)$ & $0.3172(2)$ & $0.3021(3)$ & $0.054(1)$ & $0.066(2)$ & $0.050(1)$ & $-0.005(1)$ & $0.014(1)$ & $-0.005(1)$ \\
\hline $\mathrm{O}(11)$ & $4 e$ & $0.6162(3)$ & $0.3033(2)$ & $0.9655(4)$ & $0.066(2)$ & $0.095(2)$ & $0.085(2)$ & $0.001(2)$ & $0.037(2)$ & $-0.016(2)$ \\
\hline $\mathrm{O}(12)$ & $4 e$ & $0.6658(2)$ & $0.8951(1)$ & $0.9956(4)$ & $0.049(1)$ & $0.063(2)$ & $0.085(2)$ & $-0.004(1)$ & $0.033(1)$ & $0.003(1)$ \\
\hline $\mathrm{C}(1)$ & $4 e$ & $-0.1981(3)$ & $0.0890(1)$ & $0.1430(3)$ & $0.033(1)$ & $0.026(1)$ & $0.029(1)$ & $0.003(1)$ & $0.011(1)$ & $0.002(1)$ \\
\hline $\mathrm{C}(3)$ & $4 e$ & $0.2186(2)$ & $0.0802(1)$ & $0.3070(3)$ & $0.029(1)$ & $0.029(1)$ & $0.032(1)$ & $-0.001(1)$ & $0.007(1)$ & $0.000(1)$ \\
\hline $\mathrm{C}(4)$ & $4 e$ & $0.3379(4)$ & $0.0630(2)$ & $0.4546(4)$ & $0.062(2)$ & $0.042(2)$ & $0.054(2)$ & $0.012(2)$ & $-0.017(2)$ & $-0.006(2)$ \\
\hline $\mathrm{C}(5)$ & $4 e$ & $0.0133(2)$ & $0.1419(1)$ & $-0.2262(3)$ & $0.029(1)$ & $0.023(1)$ & $0.027(1)$ & $0.0015(9)$ & $0.009(1)$ & 0.001(1) \\
\hline$C(6)$ & $4 e$ & $0.0032(4)$ & $0.0874(1)$ & $-0.3567(3)$ & $0.091(2)$ & $0.029(1)$ & $0.033(2)$ & $-0.003(1)$ & $0.030(2)$ & $-0.003(1)$ \\
\hline
\end{tabular}

Acknowledgments. The authors thank the Education Office of Hubei Province, P. R. China, for the research grant No. 2002B29002 and the Natural Science Foundation of Hubei Province, P. R. China, for the research grant No. 2003ABB010.

\section{References}

1. Sheldrick, G. M.: Siemens SHELXTL (Version 5.0). Siemens Industrial Automation, Inc., Analytical Instrumentation, USA 1995.

2. Sheldrick, G. M.: SHELXTL-plus. Release 4.1. Siemens Analytical X-ray Instruments Inc., Madison Wisconsin, USA 1991. 